\title{
GEOMATICS FOR SMART CITIES: OBTAINING THE URBAN PLANNING BAF INDEX FROM EXISTING DIGITAL MAPS
}

\author{
V. Casella ${ }^{\mathrm{a}, *}$, M. Franzini ${ }^{\mathrm{a}}$, R. De Lotto ${ }^{\mathrm{a}}$ \\ a Department of Civil Engineering and Architecture, University of Pavia, Italy \\ (vittorio.casella, marica.franzini, roberto.delotto)@unipv.it
}

ThS 17

KEY WORDS: geo-information, urban planning, geoprocessing, BAF, biotope area factor, smart city, urban analytics

\begin{abstract}
:
The urban analytics expression is spreading out. To our understanding, it deals with the capability of measuring cities and their communities, as a support to their effective planning and management. In other words, being an analytically well-known city is a precondition for pursuing smartness. Urban planning is a very important item for city management and is interrelated with many layers, including urban environmental quality, air quality and well-being. Effective urban planning is based on the knowledge of quantitative parameters such as the biotope area factor (BAF), which was originally proposed for the city of Berlin and is currently used in other cities. The BAF index is used to evaluate the degree of soil permeability and measures, to a certain extent and from a specific point of view, how a city is eco-friendly. The usual way of evaluating the BAF is based on the manual construction of dedicated maps, using existing orthophotos and oblique imagery as a support. But this method is expensive, time-consuming and non-objective, as it is prone to different interpretations. The paper presents a newly-developed methodology for calculating the BAF. It is based on the use of existing digital cartography and on the application of geoprocessing techniques from GIS science: it is therefore fully automated and objective. The Pavia city (Northern Italy) is used as a testsite and a careful validation of the developed methodology is carried out, by comparison to 12 manually surveyed test areas, corresponding to $5 \%$ of the built-up areas of the municipality.
\end{abstract}

\section{INTRODUCTION}

The urban analytics expression is spreading out. To our understanding, it deals with the capability of measuring cities and their communities, as a support to their effective planning and management. In other words, being an analytically well-known city is a precondition for pursuing smartness.

Spatial enablement is one of the engines of current development in urban analytics. Indeed many disciplines seem to have discovered only recently how much beneficial can be adding the spatial reference to data which traditionally have a pure tabular form (Angelidou, 2014; Daniel, 2013; Roche, 2014; Roche, 2015). This is probably one of the current great challenges for the community of geographic information: to promote spatial enablement (and the related contamination between traditionally separated disciplines) rather than chasing evolution.

Urban planning is a very important item for city management and is interrelated with many other layers, including urban environmental quality, air quality and well-being. Effective urban planning is based on the knowledge of quantitative parameters such as the biotope area factor (BAF), which was originally proposed for the city of Berlin and is currently used in other cities. The BAF index is used to evaluate the degree of soil permeability and measures, to a certain extent and from a specific point of view, how much eco-friendly is a city.

The usual way of evaluating the BAF is based on the manual construction of dedicated maps, using existing orthophotos and oblique imagery as a support. But this method is expensive, timeconsuming and non-objective, as it is prone to different interpretations. The paper presents a newly-developed methodology for calculating the BAF. It is based on the use of existing digital cartography and on the application of geoprocessing techniques from GIS science: it is therefore fully automated and objective. The proposed methodology shows that a non-traditional use of existing digital maps can provide new and very valuable information layers and boosts significant sectors of urban analytics. The proposed methodology is applied to the cartography of the municipality of Pavia, in Northern Italy; it is also carefully validated, by comparison with several, manually surveyed, check areas.

The authors already published a paper (De Lotto et alii, 2015) illustrating the BAF and its significance for urban planning, and reporting a detailed comparison of the traditional and novel way of estimating it. Current paper mainly deals with the geomatic aspects and accounts for new findings in the assessment of the proposed methodology. Nevertheless, the paper starts with an illustration of the BAF index, in Sec. 2, as it is necessary to understand the following part of the paper. An overview is given of the structure of the Pavia's digital cartography, in Sec. 3. Noticeably, that cartography is conformal to a regional standard, thus making our proposed methodology widely replicable. The M4BAF (Maps for BAF) methodology is illustrated in Sec. 4, while Sec. 5 shows how the novel method has been validated. Further sections are very traditional: discussion, conclusions and further activities.

\section{THE BIOTOPE AREA FACTOR DEFINITION}

Although it is not the subject of the note it is useful to provide some brief information about the biotope area factor (BAF); more can be found in (De Lotto et alii, 2015).

\footnotetext{
* Corresponding author
} 
The BAF index measures the capability of a part of a territory to recirculate the rainwater before it is collected by the urban drainage systems. Water re-entering takes place through two main mechanisms: its absorption by topsoil and percolation towards subsoil and the re-introduction into the atmosphere by the plants through the evapotranspiration mechanism.

One of the most negative consequence of buildings and roads construction is the increasing of soil sealing. The rainwater rapidly flows towards the waterways or drainage systems instead of being absorbed from the soil. During strong weather phenomena, the drainage systems are sometimes unable of absorbing the whole amount of rainfall; the excess water could cause overflows, floods, landslides and mudflows. In ordinary conditions, the decreased permeability of the soil causes the increase of air temperature, the increase of the convective air currents and the increase of the transportation of the pollution generated at ground level to the atmosphere. Finally, an impervious area is obviously unfriendly for flora and fauna.

For all the above-listed reasons, city planners, policy makers and municipality administrators are very interested in tools and indices which are able to analytically quantify soil permeability and therefore several cities have defined indices related to this aspect as a support for an effective planning.

The BAF index was introduced in 1994 by the city of Berlin (the website is referenced in the bibliography); it is based on an lookup table partly exemplified by Figure 1. It considers 9 different surface types and associates them a weighting factor between 1 (complete permeability) to 0 (complete imperviousness).

BAF calculation can be performed for any part of a city: a block, a district or the whole city. Conceptually, the considered territory, which is in general characterized by various soil typologies, must be subdivided into smaller polygons which are homogeneous in terms of the BAF coefficient. For each of these polygons the ecologically effective surface area (EESA) must be determined by multiplying the area of the polygon $\left(A_{i}\right)$ by the corresponding BAF coefficient $\left(w_{i}\right)$. The BAF value for the considered region equals the sum of all the EESAs divided by the sum of the areas, as shown by formula (1).

$$
B A F=\frac{\sum_{i=1}^{n} A_{i} w_{i}}{\sum_{i=1}^{n} A_{i}}
$$

It could be questioned whether an index defined for the city of Berlin is applicable to Lombardy and in particular to the city of Pavia. Firstly, the areas of Berlin and Pavia are reasonably comparable, in terms of climates issues and building typologies. Then the BAF abacus is quite schematic and mainly based on surface perviousness: we hardly expect significant differences from its application to the considered cities. Finally, and mostly important, we mainly are looking for a relative index, rather than absolute, allowing us to compare two districts of the same city or the same district, before and after a regeneration. For such tasks, $\mathrm{BAF}$ is perfectly fitted, at least for northern Italy.

The BAF quantification is normally carried out manually and for limited extents of the territory. An existing map (vector map, raster map or orthophoto) is used as background and a new dedicated thematic map is created, with any GIS or CAD application. Such map is constituted by polygons which are homogeneous with respect the BAF index and are classified accordingly. For this decomposition and classification task, various information sources can be used: aerial imagery, orthophotos, digital globes, oblique imagery and also visual inspections. Finally, the total area of the polygons belonging to the various classes is calculated and formula (1) is applied.

The described procedure is time consuming and prone to interpretation mistakes, this showing the need for an automated and objective way of constructing BAF maps.

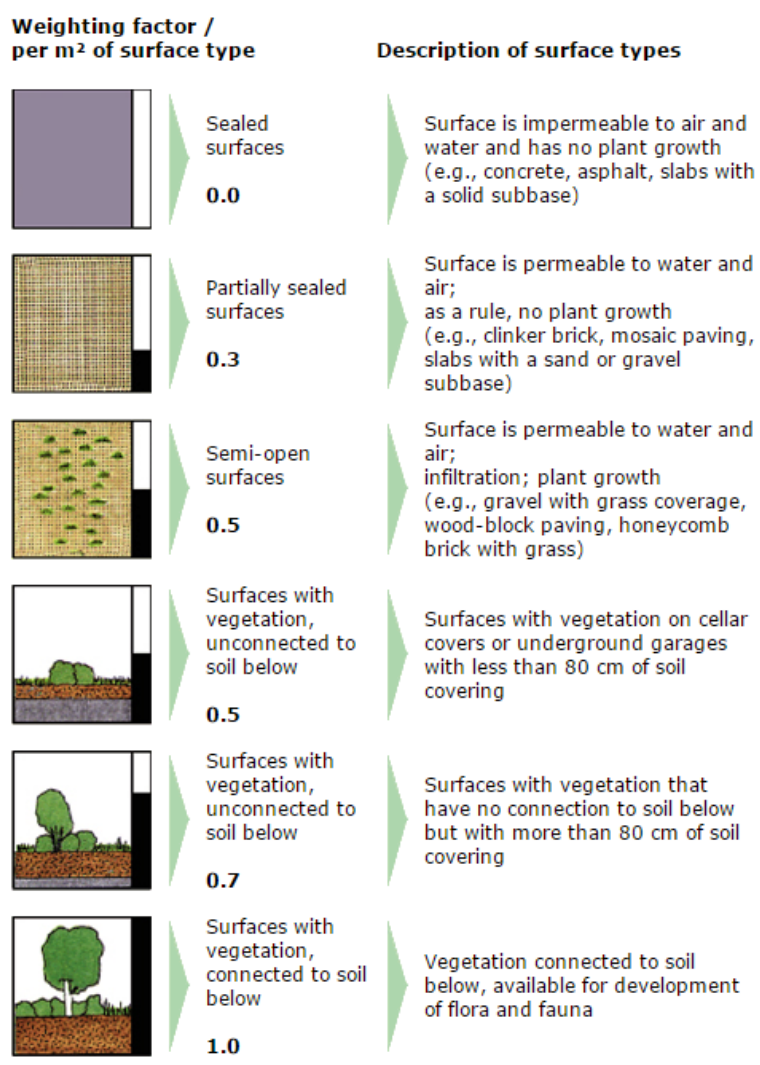

Figure 1. Excerpt from the original BAF look-up table

\section{STRUCTURE OF PAVIA'S DIGITAL MAP}

Pavia is a medium sized city having 72,000 inhabitants and an area of 63 square kilometres; it is located in Northern Italy, 35 $\mathrm{km}$ South of Milan (Figure 2). The currently used city map is owned by the Municipality and was created in the early eighties as a paper map having the 1:2,000 ratio scale.

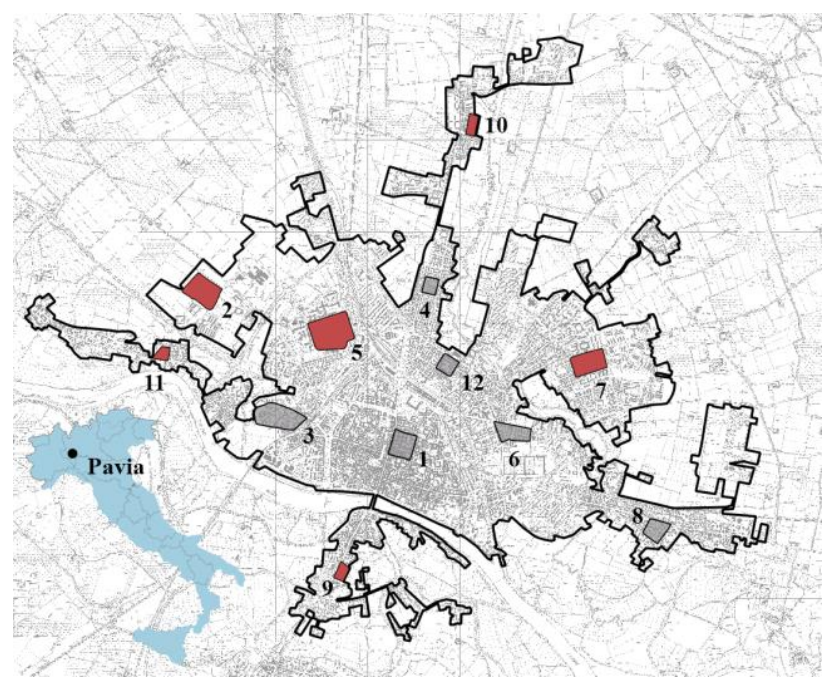

Figure 2. Location of Pavia's city in Italy; the 12 test area and the 6 for which results are presented here, in red 
The map was successively digitized and progressively updated and transformed according to the guidelines issued by the Lombardy regional Government. The map can be currently visualized at (Pavia-1) and can also be downloaded at (Pavia-2). For the present research, we had the whole map in ESRI shape format directly from the Municipality, which is here acknowledged.

As anticipated, the Lombardy Region published guidelines (which can be downloaded at (RL-1) and (RL-2)) for the creation and updating of the municipalities' large scale maps. Noticeably, the Lombardy Region has co-financed the creation from scratch of new maps or the updating of existing ones, in order to stimulate the Municipalities to adopt the regional specifications. Up to now, 1,244 municipalities out of $1,544(80 \%)$ have an updated and conformal map, according to (RL-3).

Interestingly, the regional guidelines are inspired to a sort of national standard, which is published at (Intesa-GIS). The Regions had the power to integrate the national standard by adding details, but leaving the general design untouched. In summary, Pavia's map is a good representative of the large-scale maps which are owned by many Italian municipalities.

Pavia's map is stored in an ESRI geodatabase and is preferably exported in the ESRI shape format. It consists of 74 layers of the punctual, linear or areal type. The primary key is 6-digits long, is composed by three two-digit subkeys and has a tree structure. The first two digits can take the values shown in Table 1 below, together with their meaning.

\begin{tabular}{|l|l|}
\hline $\mathbf{0 0}$ & $\begin{array}{l}\text { Geodetic, cartographic and photogrammetric } \\
\text { information }\end{array}$ \\
\hline $\mathbf{0 1}$ & Roads, mobility and transport \\
\hline $\mathbf{0 2}$ & Buildings and man-made features \\
\hline $\mathbf{0 3}$ & Street names and house numbers \\
\hline $\mathbf{0 4}$ & Hydrography \\
\hline $\mathbf{0 5}$ & Orography \\
\hline $\mathbf{0 6}$ & Vegetation \\
\hline $\mathbf{0 7}$ & Technological networks \\
\hline $\mathbf{0 8}$ & Toponyms \\
\hline $\mathbf{0 9}$ & Administrative borders \\
\hline
\end{tabular}

Table 1. The first level of the map tree-structured classification

Once chosen the ' 02 ' branch, five further classes are planned, which are listed in Table 2. Finally, five more options are available for the '0201' branch, which are listed in Table 3.

\begin{tabular}{|l|l|}
\hline 02 01 & Constructions \\
\hline 02 02 & Small man-made structures \\
\hline 02 03 & Transportation infrastructures \\
\hline 02 04 & Retaining and soil conservation structures \\
\hline 02 05 & Hydraulic constructions \\
\hline
\end{tabular}

Table 2. The admissible values for the second level of the ' 02 ' branch

\begin{tabular}{|l|l|}
\hline 02 01 01 & Volumetric unit \\
\hline 02 01 02 & Buildings \\
\hline 02 01 04 & Roofs \\
\hline 02 01 05 & Architectonic details \\
\hline 02 01 06 & Minor buildings \\
\hline
\end{tabular}

Table 3. The admissible values for the third level of the ' 0201 ' branch

For some categories, there is a secondary key for a better specification of the features' nature; the name of the secondary key and its allowable values change from one category to another. For the 020101 (Buildings) primary key value, the secondary key is named EDIFC_TY and can take a tenth of values. A second interesting example is related to a feature type named Vehicle circulation areas having 010101 as primary key value; its secondary key is named AC_VEI_FON and allows the user to distinguish between: asphalt, cement, stones, gravel, slabs of stone, pavè, ground, grass, opus incertum, and interlocking paving bricks.

In summary, the map has a very detailed classification of its content which can be used to effectively associate a BAF coefficient to each map's feature. Figure 3 shows test area 7; colours don't have any particular meaning but simply differentiate different categories, so that many colours means rich and detailed classification.

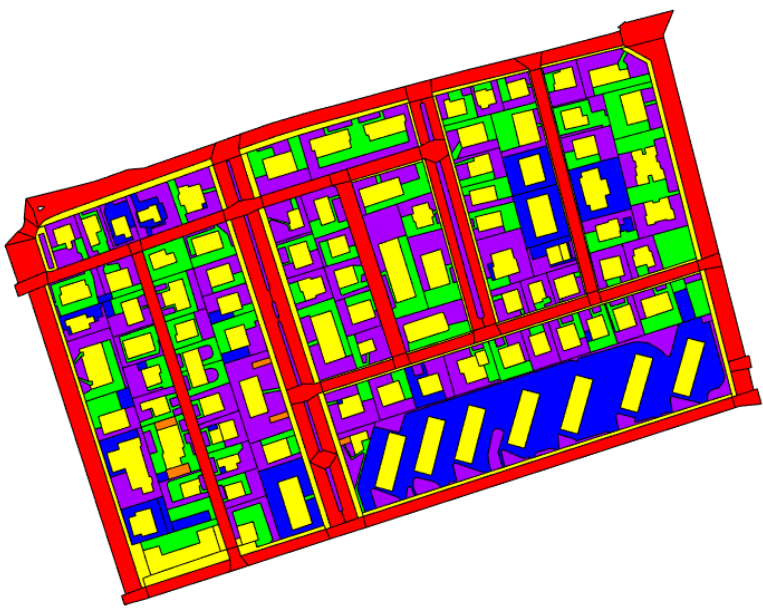

Figure 3. Illustration of the classification detail for test area 7: the various colours correspond to different categories

Nevertheless, the map presents some issues which were accurately tackled in our work. First of all, there are overlapping features such as overpassed roads, courtyards and ramps, as well as courtyards and underground garages. Moreover, courtyards are classified in a less detailed way than other features and the nature of the paving (ground, gravel, cement and pavement) is not accounted for.

\section{THE DEVELOPED METHODOLOGY}

As anticipated in the previous section, our developed methodology is based on the observation that the existing digital map of the city of Pavia is sufficiently detailed (with respect to geometry and classification) to perform a reliable BAF mapping.

The final product of our approach is a simple surface made of adjacent, non-overlapping polygons, without holes, covering all the city and giving the BAF values for every location.

First of all, a selection was performed among the existing map layers to keep only those really interesting for BAF evaluation: punctual and linear features were discarded, as well as unnecessary or redundant polygonal ones. In conclusion, a set of 29 layers, out of 74, were identified, stored in the ESRI shape format and used for subsequent analysis.

All the editing and processing work was performed with QGIS. Implementation details are omitted in the present publication and will be presented in a further one. A grand-key was added to each feature, coming from the concatenation of primary and secondary keys. A look-up table was created associating each value of the grand-key with the corresponding BAF code (between 1 and 9) and weight factor (having values between 0 and 1 , see Figure 1). 


\begin{tabular}{|l|c|l|c|r|l|}
\hline Grand-key & Key1 & Key2 & BAF code & BAF weight $(w)$ & Description \\
\hline $\mathbf{0 1 0 1 0 1 ~ 0 2 2 1}$ & 010101 & 0221 & 1 & 0 & asphalt \\
\hline $\mathbf{0 1 0 1 0 1 ~ 0 2 2 8}$ & 010101 & 0228 & 2 & 0.3 & pavè \\
\hline $\mathbf{0 1 0 1 0 1 0 2 1 2}$ & 010101 & 0212 & 6 & 1 & grass \\
\hline $\mathbf{0 2 0 2 0 4 0 1 0 2}$ & 020204 & 0102 & 6 & 1 & soccer field \\
\hline $\mathbf{0 2 0 2 0 4 0 1 0 3}$ & 020204 & 0103 & 2 & 0.3 & tennis court \\
\hline $\mathbf{0 5 0 3 9 3 ~ 0 1 0 5}$ & 050393 & 0105 & 3 & 0.5 & gravel \\
\hline $\mathbf{0 5 0 3 9 3 0 1 1 6}$ & 050393 & 0116 & 1 & 1 & sand \\
\hline
\end{tabular}

Table 4. An excerpt of the prepared look-up table

This table has 175 rows, meaning that as many categories are significant for BAF calculation; an excerpt of the table created is shown by Table 4, for the sake of clarity.

The look-up table was used to associate BAF code and weight factor to each feature of each considered layer. Then all the layers were merged to form a unique large topographic database containing all the classified features. Furthermore, overlapping polygons were taken into consideration and properly processed, as they generally have different BAF coefficients: courtyards and underground garages for instance. The layers were transformed by a complex application of various Boolean polygon operators: multi-part polygons were converted to single-part ones; two partial layers were created, that of non-overlapping polygons (or part of polygons) and that of overlapping parts of polygons; the latter was further transformed by keeping, among the multiple overlapping sub-polygons, the one with the lowest BAF coefficient, in order to be conservative; the so-transformed partial layers were finally merged to form the final BAF map. Figure 4 and Figure 5 show examples of the obtained map, in which the colour is now related with the BAF coefficient, as pointed out by the legend.

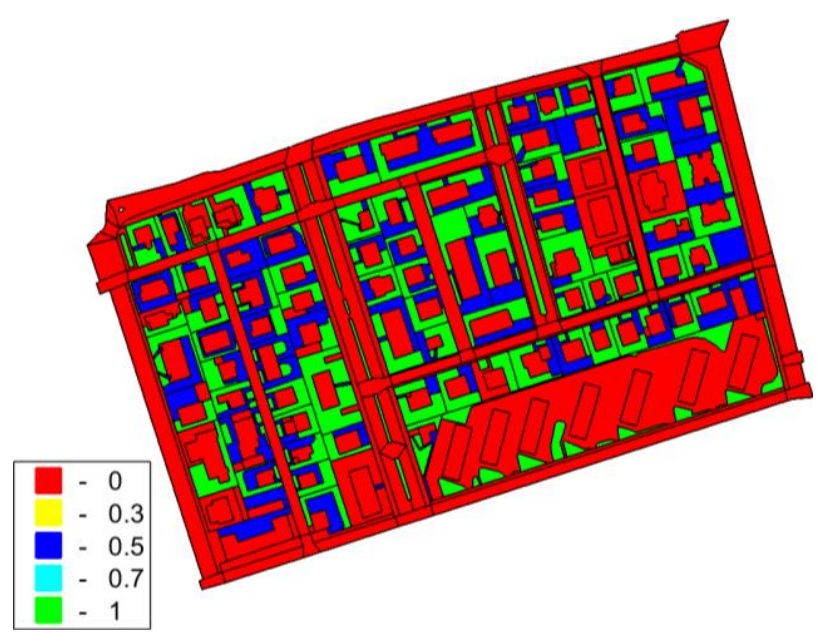

Figure 4. BAF classification for test area 7
Finally, a report procedure was created to calculate the summarized BAF values for a certain area, producing a table like Table 5, which refers to test area 7 and shows: in col. 1 the BAF coefficient considered; in col. 2 the number of features having a defined BAF value; in col. 3 the total area of the features; in col. 4 the corresponding percentage, with respect to the total area; in col. 5 the BAF equivalent area (col. 1 times col. 3); in col. 6 the corresponding percentage value. The last row summarizes results and shows the test area name, the total number of features, their total area, the total equivalent area and, finally, the BAF value for the whole area considered, coming from the ratio between the two previous values.

The reporting procedure can run in a three-fold way, as it can be applied: to the whole city map; to a partial file, obtained by cutting the general one, as we did for test areas; to a limited region of the global map, which can be interactively chosen by the user through a selection.

\section{METHODOLOGY ASSESSMENT}

The developed procedure was assessed, in order to check its capability of properly estimating the BAF value. To this goal, twelve test areas were chosen, characterized by different building typologies, whose total area roughly equals $5 \%$ of the overall built up area of the municipality of Pavia. Test areas are shown in Figure 2 as darker polygons over the background of the city map; the thick line encloses the built-up area; red areas are only reported in present paper.

For each test area, three kind of maps were prepared, which are listed and explained below.

Level 1 - The map as it is. The same map (same geometries, same classification) which can be downloaded from the web site of the Municipality; the associated tables were simply enriched with fields related to BAF classification.

Level 2 - The map as it should be. Level-1 maps could contain mistakes in polygon drawing or in feature classification, which are fixed in Level 2, according to the regional Guidelines (RL-1 and RL-2).

\begin{tabular}{|l|r|r|r|r|r|r|}
\hline BAF weight (w) & \# feature & Area [sqm] & Area [\%] & EESA[sqm] & EESA[\%] & BAF \\
\hline $\mathbf{1 . 0}$ & 169 & 19520 & 21.95 & 19520 & 75.96 & \\
\hline $\mathbf{0 . 7}$ & 0 & 0 & 0.00 & 0.00 & 0.00 & \\
\hline $\mathbf{0 . 5}$ & 105 & 12352 & 13.89 & 6176 & 24.04 & \\
\hline $\mathbf{0 . 3}$ & 0 & 0.00 & 0.00 & 0.00 & 0.00 & \\
\hline $\mathbf{0 . 0}$ & 269 & 57047 & 64.16 & 0.00 & 0.00 & \\
\hline Area 7 & $\mathbf{5 4 3}$ & $\mathbf{8 8 9 1 9}$ & & $\mathbf{2 5 6 9 6}$ & & $\mathbf{0 . 2 9}$ \\
\hline
\end{tabular}

Table 5. BAF calculation for test area 7 


\begin{tabular}{|l|r|c|c|c|c|c|}
\hline & Area [sqm] & BAF 1 & BAF 2 & BAF 3 & $\begin{array}{c}\text { Abs(delta 1-3) } \\
{[\%]}\end{array}$ & $\begin{array}{c}\text { Abs(delta 2-3) } \\
{[\%]}\end{array}$ \\
\hline Test Area 2 & 102922 & 0.53 & 0.53 & 0.49 & 7.43 & 7.43 \\
\hline Test Area 5 & 157242 & 0.48 & 0.47 & 0.47 & 0.40 & 0.00 \\
\hline Test Area 7 & 88919 & 0.29 & 0.28 & 0.26 & 9.92 & 8.37 \\
\hline Test Area 9 & 45071 & 0.65 & 0.66 & 0.66 & 0.82 & 0.00 \\
\hline Test Area 10 & 30136 & 0.35 & 0.39 & 0.39 & 8.99 & 0.12 \\
\hline Test Area 11 & 21871 & 0.28 & 0.28 & 0.27 & 4.75 & 2.28 \\
\hline Total & $\mathbf{4 4 6 1 6 2}$ & $\mathbf{0 . 4 5}$ & $\mathbf{0 . 4 5}$ & $\mathbf{0 . 4 4}$ & $\mathbf{4 . 7 5}$ & $\mathbf{3 . 5 0}$ \\
\hline
\end{tabular}

Table 6. Final results for the six test areas and for all the three levels considered

Level 3 - The true BAF map. Level-2 maps could be different from the ideal BAF maps not for compilation mistakes, but for specific aspects of the Guidelines. For instance, courtyards (which are considered less important than buildings and roads from a pure cartographic point of view) must be represented as a unique polygon even if they contain a grass part and a paved one. Thus mixture courtyards, although correctly represented with respect to the map's specifications, could compromise a good BAF calculation. To overcome these issues, Level-3 maps were created by enriching Level-2 ones, splitting existing polygons and manually assigning BAF codes and coefficients. Level-3 maps were considered as the true BAF maps. Level-2 and -3 maps were created by manually editing the existing cartography. To properly carry out this step the existing digital orthophoto of the Municipality (having a ground resolution of $10 \mathrm{~cm}$ roughly) was used as a support, as well as the various information sources which are available on the web (Google Street View, the Bing Maps oblique imagery). Finally, an on-the-field check was performed.

Table 6 shows final results for the six test areas considered here (as the remaining ones are still under the final in situ check) and for the three considered levels. Column 2 shows the area (extent measurement) of each test area; column 3 to 5 report the average $\mathrm{BAF}$ value for the considered test area and for the three depicted levels; columns 6 and 7 shows the absolute value of the difference between the BAF level 1 and 3 and BAF level 2 and 3 , respectively, in percentage units. Last row shows results for all the considered test areas, which were obtained by averaging the figures above and using the area as weight.

\section{DISCUSSION}

Figures in the $B A F 3$ column (in Table 6) refers to level-3 maps and must be considered as true. $B A F 1$ column reports values calculated on level-1 map (the current municipal map), and $B A F$ 2 is related to the level-2 map, which was amended from possibly existing mistakes.

BAF variations between levels 1 and 2 are really limited: the highest absolute difference is 0.04 , corresponding to $10 \%$ in relative terms. We can therefore conclude that Pavia's map is well done. The comparison between BAF 1 and BAF 3 figures brings to an overall percentage difference of $4.8 \%$ which is very low and highlights that BAF values can be calculated by existing digital maps with a very good quality.

\section{CONCLUSIONS}

A novel methodology has been proposed to measure the BAF value for a municipality or a part of it. It is based on performing sophisticated analysis on the city's digital map.

The methodology is automatic, objective and proved to be very accurate. The pro-posed methodology is significantly replicable as it can be applied to almost all municipalities of the Lombardy region. It could also be applied to most of the Italian cities with minor adaptations.

The proposed methodology is feasible because the considered digital map is very detailed, in terms of geometry and classification. Its replicability outside Italy depends on the characteristics and the degree of detail of the maps used in the various countries.
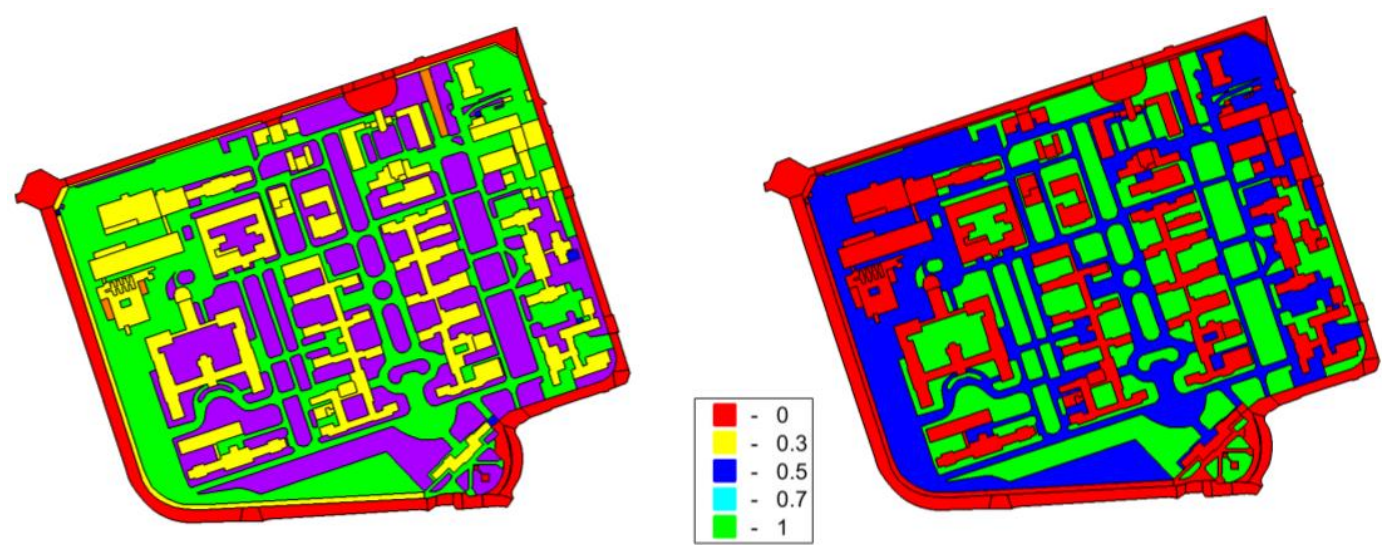

Figure 5. The original and BAF maps for test area 5 
Our described experience could also be used in a reverse way, to highlight which requirements would be necessary for a map to be a good support for BAF evaluation.

\section{FURTHER ACTIVITIES}

On-the-field verification for six remaining test areas is close to conclusion. After final validation and documentation, the developed methodology will be used by some of the authors (the urban planners) to perform detailed urban analyses concerning Pavia's city and possibly other sites. The other authors, which are active in Geomatics, meanwhile activated a collaboration with hydraulic Engineers as it seems that a similar methodology could be of great benefit for drainage system planning. Finally, this expression showed that Matlab can be a powerful geoprocessing tool: the developed code will be cleaned-up, documented and shared.

\section{ACKNOWLEDGEMENTS}

The authors gave equal contributions to the preparation of present paper. Vittorio Casella developed the classification methodology described in Sec. 4 and coded the related Matlab programs; furthermore, he conceived the assessment methodology illustrated in Sec. 5. Vittorio Casella and Marica Franzini jointly performed the analysis described and prepared tables and figures. Giuseppe Girone and Paolo Marchese (technicians working at the Geomatics Laboratory at the University of Pavia) made an impressive editing work by preparing level 2 and 3 maps. Paolo Marchese also prepared the look-up table described in Sec. 4. The Municipality of Pavia is gratefully acknowledged here for providing the maps and authorizing their use for research purposes.

\section{REFERENCES}

Akbari, H., 2002. Shade trees reduce building energy use and $\mathrm{CO} 2$ emission from power plants. Environmental Pollution, 116: 119-126.

Allen, R.G., Pereira, L.S., Raes, D., Smith, M., 1998. Crop evapotranspiration - Guidelines for computing crop water requirements. FAO Irrigation and drainage paper 56, FAO Food and Agriculture Organization of the United Nations, Rome.

Angelidou, M., 2014. Smart city policies: A spatial approach. Cities, 41, S3-S11.

Bregha, F., 1998. The Integration of Environmental Factors in Government Policy. Canadian Environmental Assessment, Research Council.

Camagni, R., Capello, R., Nijkamp, P., 1998. Towards sustainable city policy: an economy-environment technology nexus. Ecological Economics, 24: 103-118.

Daniel, S., Doran, M. A., 2013. GeoSmartCity: geomatics contribution to the Smart City. In: Proceedings of the 14th Annual International Conference on Digital Government Research (pp. 65-71). ACM.

De Lotto, R., Casella, V., Franzini, M., Gazzola, V., Morelli di Popolo, C., Sturla, S., Venco, E.M., 2015. Estimating the Biotope
Area Factor (BAF) by Means of Existing Digital Maps and GIS Technology. Computational Science and Its Applications-ICCSA 2015, Springer International Publishing: 617-632.

De Lotto, R., 2008. Assessment of development and regeneration urban projects: cultural and operational implications in metropolization context. International Journal of Energy and Environment 1, vol. 2:24-35.

European Commission Strategic Environmental Assessment (SEA) http://ec.europa.eu/environment/funding/cei_en.htm. Last access on 22.04.2016.

Hargreaves, G.H., Samani, Z.A., 1982. Estimating potential evapotranspiration - Tech. Note. Journal of Irrigation and. Drain Engineering, ASCE 108.

Kruse, A., 2011. GRaBS Expert Paper 6: The Green Space Factor and the Green Points System, GRaB. The GRaBS project. London: Town and Country Planning Association \& GRaBS .

Lakes, T., Kim, H. O., 2012. The urban environmental indicator "Biotope Area Ratio"- An enhanced approach to assess and manage the urban ecosystem services using high resolution remote-sensing. Ecological Indicators, 13(1): 93-103.

National Technical Standard for Topographic Database, http://tinyurl.com/jakrlyr. Last access on 22.04.2016 [Referenced as Intesa-GIS]

Pavia-1 - WebGIS of the Cartography of Pavia, http://tinyurl.com/hjx2g7x. Last access on 22.04.2016.

Pavia-2 - Download page of the cartography of the Pavia, http://tinyurl.com/hj3zrjm. Last access on 22.04.2016.

RL-1 - Regione Lombardia - Guidelines for the Topographic Database - Content Specifications, http://tinyurl.com/jasblhj. Last access on 22.04.2016.

RL-2 - Regione Lombardia - Guidelines for the Topographic Database - Annex. A - Content Specifications, http://tinyurl.com/hq64e9h. Last access on 22.04.2016.

RL-3 - Regione Lombardia - Advancement of the Topographic Database Project, http://tinyurl.com/hb567ye. Last access on 22.04.2016.

Roche, S., 2014. Geographic Information Science I Why does a smart city need to be spatially enabled?. Progress in Human Geography, 38(5), 703-711.

Roche, S., 2015. Geographic information science II Less space, more places in smart cities. Progress in Human Geography, 0309132515586296.

Senate Department of Urban Development and the Environment (Berlin) http://tinyurl.com/zree5hu. Last access on 22.04.2016.

Steiner, F., 2000. The living landscape: an ecological approach to Landscape Planning. McGraw Hill Professional, Milano. 